\title{
Assessment of Thromboembolic Risk of Cessation of Oral Anticoagulation Post Catheter Ablation in Patients with and without Atrial Fibrillation Recurrence
}

\author{
Bing Rong${ }^{1}$, Wenqiang Han ${ }^{1}$, Mingjie Lin $^{1}$, Li Hao ${ }^{2}$, Kai Zhang ${ }^{1}$, Tongshuai Chen ${ }^{1}$, Rina \\ Sha ${ }^{1}$, Juntao Wang ${ }^{1}$, Rong Wang ${ }^{1}$, and Jingquan Zhong ${ }^{1}$ \\ ${ }^{1}$ Shandong University Qilu Hospital \\ ${ }^{2}$ Shandong Qianfoshan Hospital
}

July 27, 2020

\begin{abstract}
Instruction Cessation of oral anticoagulation (OAC) is common after the first 3 months of catheter ablation of atrial fibrillation (AF); however, thromboembolic risk has not been defined in patients with and without AF recurrence (RAF vs. NRAF) post ablation

Methods and Results We identified 796 patients who discontinued OAC at 3 months post AF ablation from January 2015 to May 2018 in our center. Regular follow-up was performed to detect RAF, collect medication management and thromboembolic and major bleeding events. $\mathrm{CHA}_{2} \mathrm{DS}_{2}$-VASc score was $1.79 \pm 1.50 ; 547$ (68.7\%) patients were at intermediate and high risk (i.e. $\mathrm{CHA}_{2} \mathrm{DS}_{2}$-VASc score ${ }^{3} 1$ in male patients, or ${ }^{3} 2$ in female patients); 169 (21.2\%) were RAF. During $29.2 \pm 12.2$ months followup, the incidence rate of thromboembolism was 1.62 per 100 patient-year (7 in 431 years) in RAF, 0.33 per 100 patient-year (5 in 1503 years) in NRAF. After adjusting for potential confounding factors, RAF was associated with more 3.5-fold higher rate of thromboembolism compared with NRAF (adjusting HR, 4.488; 95\%CI, 1.381-14.586). Rate of thromboembolism was even higher in patients with intermediate and high risk (2.16 per 100 patient-year [7 in 323 years] versus 0.38 per 100 patient-year [4 in 1043 years], aHR, 5.807; 95\%CI, 1.631-20.671). In multivariate logistic regression analysis, RAF was the only independent predictor of thromboembolism (4.837 [1.498-15.621], $P=0.008)$.
\end{abstract}

\section{Introduction}

Atrial fibrillation (AF), the most common arrhythmia, is associated with an approximately fivefold increased risk of stroke. ${ }^{1}$ Catheter ablation is effective as rhythm control strategy in AF patients. ${ }^{2}$ Use of long-term oral anticoagulation (OAC) treatment, i.e., after the first 3 months post ablation of AF, remains controversial. Although studies have reported a low incidence rate of thromboembolism after ablation in patients discontinuing $\mathrm{OAC}$ compared with those continuing $\mathrm{OAC},{ }^{3,4}$ the thromboembolic risk has been inconsistent particularly among studies including patients with and without AF recurrence. ${ }^{5-8}$ Cessation of OAC is common ( from $30 \%$ to 95\%) in AF patients undergoing or not undergoing catheter ablation, ${ }^{3,9-12}$ although guidelines have recommended that $\mathrm{OAC}$ should follow general anticoagulation recommendations regardless of the presumed rhythm outcome after catheter ablation. ${ }^{1,13,14}$ The diversity of approaches in clinical practice might be secondary to low level evidence and absence of data about the risk of thromboembolism.

In patients with versus those without $\mathrm{AF}$ recurrence (RAF vs. NRAF) after ablation, the incidence rate and hazard risk of thromboembolism after OAC discontinuation remains unclear. We therefore reviewed data on efficacy and complications after catheter ablation at our center to investigate the incidence rate 
of thromboembolic events and to identify the risk factors for thromboembolism after cessation of OAC in patients with and without AF recurrence.

\section{Methods}

This study was approved by the local institutional Ethics Committee, and written informed consent was obtained from all participants. The present analysis of events of thromboembolism and major bleeding (MB) after cessation of $\mathrm{OAC}$ at 3 months post ablation was based on data from a prospective observational study (Chinese Clinical Trial Registry: ChiCTR-OCH-14004674) with three-year follow-up of patients who underwent ablation for AF at our center. The study collected data on the efficacy of radiofrequency and cryogenic catheter ablation, pharmacotherapy, and complications including death, thromboembolism, MB and rehospitalization. All patients undergoing AF catheter ablation between January 2015 and May 2018 were consecutively included in the study unless they met any of the following exclusion criteria: (i) valvular heart disease; (ii) follow-up for $<12$ months; or (iii) thromboembolic or MB events during the first 3 months after ablation.

Baseline data were collected before procedure. $\mathrm{CHA}_{2} \mathrm{DS}_{2}$-VASc score was used to stratify thromboembolic risk. $\mathrm{CHA}_{2} \mathrm{DS}_{2}$-VASc scores of 0,1 , [?] male patients and 1, 2, [?] female patients were considered to correspond to low, intermediate and high risk of thromboembolism, respectively. As recommended, ${ }^{14} \mathrm{HAS}-\mathrm{BLED}$ score was used to evaluate bleeding risk; no score points were given for use of nonsteroidal anti-inflammatory drugs (except antiplatelet drugs) or labile international normalized ratio values because of incomplete information.

\section{Postprocedural management and follow-up strategy}

Medical management during the first 3 months after ablation has been previously described. ${ }^{15}$ After discharge, patients underwent heart rhythm evaluation by electrocardiography (ECG) and 24-hour Holter monitoring at 1, 3, and 6 months and every 6 months thereafter. If the patient did not show up for scheduled follow-up monitoring visits, within one month our follow-up office would call the patient to collect information on recurrence and complications. Atrial tachyarrhythmia recurrence was defined as developing atrial tachyarrhythmia lasting at least 30 seconds after the 3-months observation window after ablation.

After the 3-months observation period post ablation, OAC use in patients without AF recurrence was determined in conjunction with the electrophysiologist; OAC use was encouraged in the high-risk RAF patients.

\section{Primary outcome events}

Study primary outcomes included rates of thromboembolism and MB events. Thromboembolism events included ischemic stroke, transient ischemic attack, and systemic embolism, which were diagnosed based on symptoms and computerized tomography or magnetic resonance imaging. MB events included intracranial hemorrhage and any other bleeding events requiring hospitalization. Timing and outcome of primary events were recorded during follow-up.

\section{Statistical analysis}

Data analysis was performed using SPSS 25.0 (IBM Corp.), and the significance level was set at $P<0.05$. The Kolmogorov-Smirnov test was used to check for normality of distribution of continuous variables, which are expressed as mean \pm standard deviation (SD) and were compared using the $t$ test or Fisher's test, while categorical variables are expressed as numbers with percentage and were compared using the chi-square test.

Crude incidence rates of thromboembolic events were expressed as rates per 100 patient-years. Cumulative survival free from thromboembolic outcome is presented as Kaplan-Meier curve. Cox hazard regression analysis was used to calculate the hazard ratio of the thromboembolic risk between RAF and NRAF groups, both overall and for intermediate- and high-risk patients. Potential confounders, including RAF, age, sex, AF type, all factors in $\mathrm{CHA}_{2} \mathrm{DS}_{2}$-VASc score, smoking, alcohol consumption, left atrium diameter [?], and obesity (body mass index[?]28.0). 
Factors associated with thromboembolism during follow-up were analyzed using uni- and multivariable logistic regression analyses in RAF and NRAF of overall or intermediate- and high-risk patients (significance level set at $P<0.10$ for inclusion into model). All factors in $\mathrm{CHA}_{2} \mathrm{DS}_{2}$-VASc score were analyzed, with age as continuous variable, and AF type, smoking, alcohol consumption, left atrium diameter [?], and obesity as categorical variables.

\section{Results}

As shown in Figure 1, of the 871 consecutive patients who underwent catheter ablation between January 2015 and May 2018, 796 patients were included in the present analysis and underwent 826 catheter ablation procedures (average 1.04 per patient). Mean $\mathrm{CHA}_{2} \mathrm{DS}_{2}$-VASc score was 1.79+-1.50; 547 (68.7\%) patients were at intermediate and high risk. Mean HAS-BLED score was $0.76+-0.76$. Follow-up duration was 29.2+12.2 months. During follow-up, all patients had at least one ECG or 24h Holter evaluation after 3 months post ablation, while only $479(60.2 \%)$ had $24 \mathrm{~h}$ Holter recording; $154(19.3 \%)$ were on antiplatelet drugs continuously, and $219(27.5 \%)$ on antiarrhythmic drugs, with the proportion being much higher in RAF versus NRAF group. Three patients died of cancer, fatal stroke, and myocardial infarction, respectively, after 12 months post ablation. $169(21.2 \%)$ patients developed atrial tachyarrhythmia after the 3-month observational period, $24(14.2 \%)$ developed persistent $\mathrm{AF}$, and 14 (8.3\%) were asymptomatic. Comparison of baseline and follow-up data between RAF and NRAF are presented in Table 1. Overall, $\mathrm{CHA}_{2} \mathrm{DS}_{2}-\mathrm{VASc}$ score was higher in RAF vs. NRAF $(P<0.05)$.

\section{Primary outcome events}

Overall, 12 patients experienced thromboembolic events (Table 2) and 1 patient suffered an MB event (subarachnoid hemorrhage in patient without AF recurrence). No patient on continuous OAC experienced a thromboembolic or MB event. The rate of sequela was numerically higher in RAF vs. NRAF (4/7 versus $1 / 5$ ). The incidence rate of thromboembolic events was 0.33 per 100 patient-year ( 5 in 1503 patient-years) in NRAF, and 1.62 per 100 patient-year (7 in 431 patient-years) in RAF. In patients at intermediate and high risk, the incidence rate of thromboembolic events was 0.38 per 100 patient-year (4 in 1043 patient-years) in NRAF, and 2.16 per 100 patient-year (7 in 323 patient-years) in RAF. Compared with NRAF, after adjusting potential confounders, RAF had significantly higher incidence rate of thromboembolic events (overall: aHR, 4.488; 95\%CI, 1.381-14.586, $P=0.013$ ) and (intermediate and high risk: aHR, 5.807; 95\%CI, 1.631-20.671, $P$ $=0.007$ ) (Table 3 and Supplementary Table S1). The Kaplan-Meier curves demonstrated higher cumulative survival rate free from thromboembolism in overall, or intermediate and high risk patients NRAF vs. RAF $(P=0.002$ and 0.001 , respectively, Figure 2$)$; events happened post 6 months after ablation, and happened post the first recording episode of recurrent AF in RAF (6/7, Table 2).

\section{Risk factors associated with thromboembolic events}

As listed in Table 4, in univariable logistic regression analysis, $\mathrm{CHA}_{2} \mathrm{DS}_{2}$-VASc score was associated with thromboembolic events in patients overall and in RAF, but not in NRAF (Supplementary Table S2). No other factors were associated with thromboembolic events in this investigation. In multivariable logistic regression analysis, after adjustment for age and sex, $\mathrm{CHA}_{2} \mathrm{DS}_{2}$-VASc score was not an independent predictor, in patients overall or in RAF (1.406 [0.920-2.147], $P=0.115$; and 1.592 [0.977-2.594], $P=0.062$ respectively). If adding $\mathrm{AF}$ recurrence as potential factor, in multivariable logistic regression analysis, AF recurrence was the strong predictor in all patients (4.837 [1.498-15.621], $P=0.008)$.

\section{Discussion}

The present study, which to the best of our knowledge is the first to compare the thromboembolic risk of cessation of OAC in RAF and NRAF after catheter ablation documented: (i) a low rate of thromboembolic events after successful catheter ablation; (ii) an approximately 3.5-fold higher incidence rate of thromboembolic events in RAF vs. NRAF; and (iii) that the $\mathrm{CHA}_{2} \mathrm{DS}_{2}$-VASc score may still predict thromboembolic risk in RAF but not in NRAF in whom OAC had been discontinued.

\section{Cessation of OAC after catheter ablation and risk of thromboembolism}


Catheter ablation has been associated with improvement in structural remodeling, hemodynamic function of the left atrium (LA) and intra-LA blood stasis in NRAF, which would decrease risk for thrombogenesis. ${ }^{16}$ Results from a large prospective real-world observational registry study of Chinese patients with $\mathrm{AF}(\mathrm{n}=4512)$ indicated that compared with patients continuing long-term OAC after successful ablation, the thromboembolic risk was low in patients discontinuing long-term OAC (0.54 vs. 0.86 per 100 patient-years), as was risk for MB events (0.19 vs. 0.35 per 100 patient-years). ${ }^{3}$ Similarly, for patients who had undergone successful ablation, Themistoclakis et al. ${ }^{4}$ reported that the incidence rate of ischemic stroke and MB events was lower in patients off OAC than in those on $\mathrm{OAC}(0.07 \%$ vs. $0.45 \%, P=0.06 ; 0.04 \%$ vs. $2 \%, P<0.0001)$. In the present study, in NRAF, the incidence rate of thromboembolism was 0.33 or 0.38 per 100 patient-year in the overall or intermediate and high risk populations. The annual incidence of stroke events for general Chinese population was 377 per 100,000 in $2013 .{ }^{17}$ Therefore, consistent with study by Yanget al. ${ }^{3}$ for NRAF, thromboembolic risk was low and similar to the general population.

Some studies have reported on thromboembolic risk for RAF vs. NRAF. Bunch et al. ${ }^{6}$ reported that across all $\mathrm{CHADS}_{2}$ scores and ages, AF patients who underwent ablation had a similar long-term risk of stroke to that of patients without AF but lower than that of patients who did not undergo ablation. Another study based on Danish administrative registries ${ }^{7}$ reported similar thromboembolic incidence rates in patients on and off OAC, even in those at high-risk $\left(\mathrm{CHA}_{2} \mathrm{DS}_{2}\right.$-VASc score [?]2). In contrast, in the Swedish catheter ablation register, ${ }^{5}$ patients with $\mathrm{CHA}_{2} \mathrm{DS}_{2}$-VASc score [?]2 and off warfarin treatment had a higher rate of ischemic stroke compared with those on warfarin treatment $(1.6 \%$ vs. $0.3 \%$ per year, $P=0.046)$. A meta-analysis in 2017 including 9 observational studies reported that the overall rate of thromboembolism was not significantly different between patients on or off OAC, however, the incidence of total bleeding events was higher among patients continuing OAC patients. ${ }^{18}$ The inconsistency in results may result from differences in race/ethnicity, proportion of complications, follow-up duration, and incidence of recurrence. Indeed, in this study, AF recurrence was significantly associated with increased thromboembolic risk in univariate model, ${ }^{7}$ and in the Swedish catheter ablation register ${ }^{5}$ or Taiwanese ablated patients, ${ }^{19} 8$ of 11 patients with ischemic stroke events had suffered AF recurrence, which may indicate that it is not proper to evaluate the thromboembolic risk in mixed patient populations with and without AF recurrence. In the present study, the incidence rate of thromboembolic events was much higher in RAF vs. NRAF; in addition to worse outcomes in AF-related strokes, the sequelae of thromboembolism were more severe in RAF. In a single-center study, the estimated 5-year stroke incidence was $3 \%$ for patients discontinuing OAC after successful ablation compared with $23 \%$ for patients with AF recurrence remaining on warfarin. ${ }^{20}$ The latter results suggest that it is reasonable to use a differential strategy in RAF vs. NRAF. Noteworthy, consistent with previous studies, ${ }^{4,5}$ in present study, all thromboembolic events happened post 6 months after ablation if cessation of $\mathrm{OAC}$, which were mostly post the time of the first recording $\mathrm{AF}$ recurrence providing the practicable space to take timely intervention of OAC. However, the temporal association between the AF episode and need for OAC still needs further confirmation.

\section{Potential factor associated with post catheter ablation thromboembolism}

Many potential factors associated with thromboembolism in general AF patients may still be suitable for predicting the risk of thromboembolism after catheter ablation, such as abnormal clotting function, low velocity of intra-atrial flow, atrial myopathy, and cardiovascular diseases. ${ }^{1,14}$ Reportedly, atrial myopathy, assessed by cardiac magnetic resonance, bipolar voltage mapping, may have a relationship with stroke. ${ }^{21}$ While in present study, because of some restrictions, we did not always record the data of bipolar voltage mapping and not regularly assess fibrosis by cardiac magnetic resonance.

$\mathrm{CHA}_{2} \mathrm{DS}_{2}$-VASc score was significantly associated with increased thromboembolic risk (Table 4), as had been previously reported. ${ }^{10}$ Of note, in the present study, $\mathrm{CHA}_{2} \mathrm{DS}_{2}$-VASc score was the only predictor of thromboembolism incidence in RAF but not in NRAF. In previous studies of patients who had undergone successful ablation or in mixed populations, previous thromboembolism was the only independent predictor among components of $\mathrm{CHA}_{2} \mathrm{DS}_{2}$-VASc score associated with thromboembolic events. ${ }^{3,5,7}$ While in multiple analysis, $\mathrm{CHA}_{2} \mathrm{DS}_{2}$-VASc score was not an strong predictor; if adding $\mathrm{AF}$ recurrence as a potential factor, 
AF recurrence was the strong predictor, which implies the performance of catheter ablation is a crucial factor for prognosis of AF patients after catheter ablation as previous publish reported (174 ablated patients, 47 +- 23 months). ${ }^{19}$

As per the latest recommendations (2019 AHA/ACC/HRS, 2017 HRS/EHRA/ECAS/APHRS /SOLAECE expert consensus, $2016 \mathrm{ESC}),{ }^{1,13,14}$ long-term continuation of OAC after 2 months post ablation should be based on the patient's stroke risk profile (i.e. $\mathrm{CHA}_{2} \mathrm{DS}_{2}$-VASc score), and not on the efficacy of ablation. However, the published results have been inconsistent which may underlie the variety of approaches seen in clinical practice. The ratio of benefits to risks for OAC and economic reasons also underlie the high proportion of cessation of OAC after the first 3 months post ablation. ${ }^{9}$ Although the present study was a single-center observational study, its results underscore the need for differential management between patients with versus those without AF recurrence after ablation, which may translate into better outcomes.

\section{Limitations}

The present study has the limitations inherent to its single-center observational design which may introduce selection bias; large-scale randomized studies are warranted to provide high-level evidence, such as the OCEAN trial. ${ }^{22}$ AF recurrence may have been underestimated because of how the events were captured during follow-up which may have affected mostly the observed incidence rate of thromboembolism in AF free patients. It would be better with extended ECG monitoring, like long-term daily life ECG or implantable loop recorders, to detect heart rhythm, build the relationship between AF burden and thromboembolic events, and improve medical care timely. ${ }^{23-25}$ Moreover, the small sample size might underlie the lack of achievement of statistical significance in multivariate analysis for $\mathrm{CHA}_{2} \mathrm{DS}_{2}$-VASc score as a predictor of thromboembolic events. Finally, missing data for the components of the HAS-BLED might have led to a lower score.

\section{Conclusions}

Discontinuation of OAC in patients who had undergone successful ablation may be reasonable with strict post-procedure monitoring; however, high level evidence is warranted. Patients who experience AF recurrence would appear to benefit from not discontinuing OAC if they have a high-risk stroke profile because of high incidence rate of thromboembolic events.

\section{References}

1. Calkins H, Hindricks G, Cappato R, et al. 2017 HRS/EHRA/ECAS/APHRS/SOLAECE expert consensus statement on catheter and surgical ablation of atrial fibrillation. Europace.2018;20(1):e1-e160.

2. Packer DL, Mark DB, Robb RA, et al. Effect of Catheter Ablation vs Antiarrhythmic Drug Therapy on Mortality, Stroke, Bleeding, and Cardiac Arrest Among Patients With Atrial Fibrillation: The CABANA Randomized Clinical Trial. JAMA. 2019;321(13):1261-1274.

3. Yang WY, Du X, Jiang C, et al. The safety of discontinuation of oral anticoagulation therapy after apparently successful atrial fibrillation ablation: a report from the Chinese Atrial Fibrillation Registry study.Europace. 2020;22(1):90-99.

4. Themistoclakis S, Corrado A, Marchlinski FE, et al. The risk of thromboembolism and need for oral anticoagulation after successful atrial fibrillation ablation. J Am Coll Cardiol.2010;55(8):735-743.

5. Sjalander S, Holmqvist F, Smith JG, et al. Assessment of Use vs Discontinuation of Oral Anticoagulation After Pulmonary Vein Isolation in Patients With Atrial Fibrillation. JAMA Cardiol.2017;2(2):146-152.

6. Bunch TJ, May HT, Bair TL, et al. Atrial fibrillation ablation patients have long-term stroke rates similar to patients without atrial fibrillation regardless of CHADS2 score. Heart Rhythm.2013;10(9):1272-1277.

7. Karasoy D, Gislason GH, Hansen J, et al. Oral anticoagulation therapy after radiofrequency ablation of atrial fibrillation and the risk of thromboembolism and serious bleeding: long-term follow-up in nationwide cohort of Denmark. Eur Heart J. 2015;36(5):307-314a. 
8. Gallo C, Battaglia A, Anselmino M, et al. Long-term events following atrial fibrillation rate control or transcatheter ablation: a multicenter observational study. J Cardiovasc Med (Hagerstown).2016;17(3):187193.

9. Bai Y, Wang YL, Shantsila A, Lip GYH. The Global Burden of Atrial Fibrillation and Stroke: A Systematic Review of the Clinical Epidemiology of Atrial Fibrillation in Asia. Chest.2017;152(4):810-820.

10. Arai M, Okumura Y, Nagashima K, et al. Adverse Clinical Events during Long-Term Follow-Up After Catheter Ablation of Atrial Fibrillation. Int Heart J. 2019;60(4):812-821.

11. Nuhrich JM, Kuck KH, Andresen D, et al. Oral anticoagulation is frequently discontinued after ablation of paroxysmal atrial fibrillation despite previous stroke: data from the German Ablation Registry. Clin Res Cardiol. 2015;104(6):463-470.

12. Noseworthy PA, Yao X, Deshmukh AJ, et al. Patterns of Anticoagulation Use and Cardioembolic Risk After Catheter Ablation for Atrial Fibrillation. J Am Heart Assoc. 2015;4(11).

13. January CT, Wann LS, Calkins H, et al. 2019 AHA/ACC/HRS Focused Update of the 2014 AHA/ACC/HRS Guideline for the Management of Patients With Atrial Fibrillation: A Report of the American College of Cardiology/American Heart Association Task Force on Clinical Practice Guidelines and the Heart Rhythm Society. J Am Coll Cardiol.2019;74(1):104-132.

14. Kirchhof P, Benussi S, Kotecha D, et al. 2016 ESC Guidelines for the management of atrial fibrillation developed in collaboration with EACTS.Eur Heart J. 2016;37(38):2893-2962.

15. Lin M, Hao L, Cao Y, et al. Successful radiofrequency catheter ablation of atrial fibrillation is associated with improvement in left ventricular energy loss and mechanics abnormalities. Int $J$ Cardiovasc Imaging. 2019;35(3):427-435.

16. Lin M, Hao L, Cao Y, et al. Successful catheter ablation of atrial fibrillation improves but not reverses the abnormalities of left atrial mechanics and energy loss. Echocardiography. 2019;36(4):752-760.

17. Guan T, Ma J, Li M, et al. Rapid transitions in the epidemiology of stroke and its risk factors in China from 2002 to 2013. Neurology. 2017;89(1):53-61.

18. Santarpia G, De Rosa S, Sabatino J, Curcio A, Indolfi C. Should We Maintain Anticoagulation after Successful Radiofrequency Catheter Ablation of Atrial Fibrillation? The Need for a Randomized Study.Front Cardiovasc Med. 2017;4:85.

19. Lin YJ, Chao TF, Tsao HM, et al. Successful catheter ablation reduces the risk of cardiovascular events in atrial fibrillation patients with CHA2DS2-VASc risk score of 1 and higher. Europace.2013;15(5):676-684.

20. Nademanee K, Schwab MC, Kosar EM, et al. Clinical outcomes of catheter substrate ablation for high-risk patients with atrial fibrillation. J Am Coll Cardiol. 2008;51(8):843-849.

21. Shen MJ, Arora R, Jalife J. Atrial Myopathy. JACC Basic Transl Sci. 2019;4(5):640-654.

22. Verma A, Ha ACT, Kirchhof P, et al. The Optimal Anti-Coagulation for Enhanced-Risk Patients Post-Catheter Ablation for Atrial Fibrillation (OCEAN) trial. Am Heart J. 2018;197:124-132.

23. Guo Y, Wang H, Zhang H, et al. Mobile Photoplethysmographic Technology to Detect Atrial Fibrillation. J Am Coll Cardiol.2019;74(19):2365-2375.

24. Guo Y, Lane DA, Wang L, et al. Mobile Health Technology to Improve Care for Patients With Atrial Fibrillation. J Am Coll Cardiol.2020;75(13):1523-1534.

25. Chen LY, Chung MK, Allen LA, et al. Atrial Fibrillation Burden: Moving Beyond Atrial Fibrillation as a Binary Entity: A Scientific Statement From the American Heart Association. Circulation.2018;137(20):e623e644. 
Table 1. Baseline and follow-up information

Baseline data

Age, mean (SD), years

65 years, $\mathrm{n}(\%)$

75 years, $\mathrm{n}(\%)$

Male, $\mathrm{n}(\%)$

Persistent AF, $\mathrm{n}(\%)$

Congestive heart failure, $\mathrm{n}(\%)$

Hypertension, $\mathrm{n}(\%)$

Diabetes mellitus, n(\%)

Prior stroke/TIA/systemic embolism, n(\%)

Vascular disease, $\mathrm{n}(\%)$

CHA2DS2-VASc score, mean (SD)

HAS-BLED score, mean (SD)

Renal disease, $\mathrm{n}(\%)$

Liver disease, $\mathrm{n}(\%)$

Anemia, $\mathrm{n}(\%)$

Alcohol consumption, $\mathrm{n}(\%)$

Body mass index, mean (SD)

LAD [?] $40 \mathrm{~mm}, \mathrm{n}$ (\%)

Follow-up data

Follow-up duration, mean(SD), months

Antiplatelet drugs, $\mathrm{n}(\%)$

Antiarrhythmic drugs, $\mathrm{n}(\%)$

ACEI/ARB, n(\%)

Statins, $\mathrm{n}(\%)$

Abbreviations: AF, atrial fibrillation; CHA2DS2-VASc, congestive heart failure, hypertension, age 75 years or older (double $\mathrm{P}<0.05$, compared with no recurrence in total patients; $+\mathrm{P}<0.05$, compared with no recurrence in intermediate or high risk

Table 2. Characteristics of patients with thromboembolic events 
Table 2. Characteristics of patients with thromboembolic events

Table 3. Univariable and multivariable Cox regression for the incidence of thromboembolic events in all patients $(\mathrm{n}=796)$

Variable

AF recurrence

Age

Female sex

Persistent AF

Congestive heart failure

Hypertension

Diabetes mellitus

Prior stroke/TIA /systemic embolism

Vascular disease

CHA2DS2-VASc score

Smoking

Alcohol consumption

LAD [?] $40 \mathrm{~mm}$

Obesity

Abbreviations: AF, atrial fibrillation; CHA2DS2-VASc, congestive heart failure, hypertension, age 75 years or older (double

Table 4. Individual risk factors associated with thromboembolism after AF ablation in all and recurrent patients

Variable

Table 4.

Variable

Age

Univaric

$\operatorname{HR}(95 \%$

Female sex

$1.043(0$.

Persistent AF

$1.178(0$.

Congestive heart failure

$0.798(0$.

Hypertension

$0.483(0$.

Diabetes mellitus

$2.193(0$.

Prior stroke/TIA /systemic embolism

$2.132(0$.

Vascular disease

2.140 (0.

CHA2DS2-VASc score

$2.542(0$.

Smoking

Alcohol consumption

$0.733(0$.

LAD [?] $40 \mathrm{~mm}$

0.899 (0.

$1.683(0$. 
\begin{tabular}{lc}
\hline Table 4. Individual risk factors associated with thromboembolism after AF ablation in all and recurrent patients & Table 4. \\
\hline Obesity & $0.935(0$.
\end{tabular}

Table 4. Individual risk factors associated with thromboembolism after AF ablation in all and recurrent patients (continuec Variable

Age

Female sex

Persistent AF

Congestive heart failure

Hypertension

Diabetes mellitus

Prior stroke/TIA /systemic embolism

Vascular disease

CHA2DS2-VASc score

Smoking

Alcohol consumption

LAD [?] $40 \mathrm{~mm}$

Obesity

Abbreviations: AF, atrial fibrillation; CHA2DS2-VASc, congestive heart failure, hypertension, age 75 years or older (double

\section{Figure legends}

Figure 1. Flowchart of study patients with atrial fibrillation. OAC, oral anticoagulation.

Figure 2. Kaplan-Meier curves for cumulative survival free from thromboembolic events in overall (A, $P$ $=0.002)$ and in intermediate and high risk $(\mathrm{B}, P=0.001)$ patient populations.

Figure 1

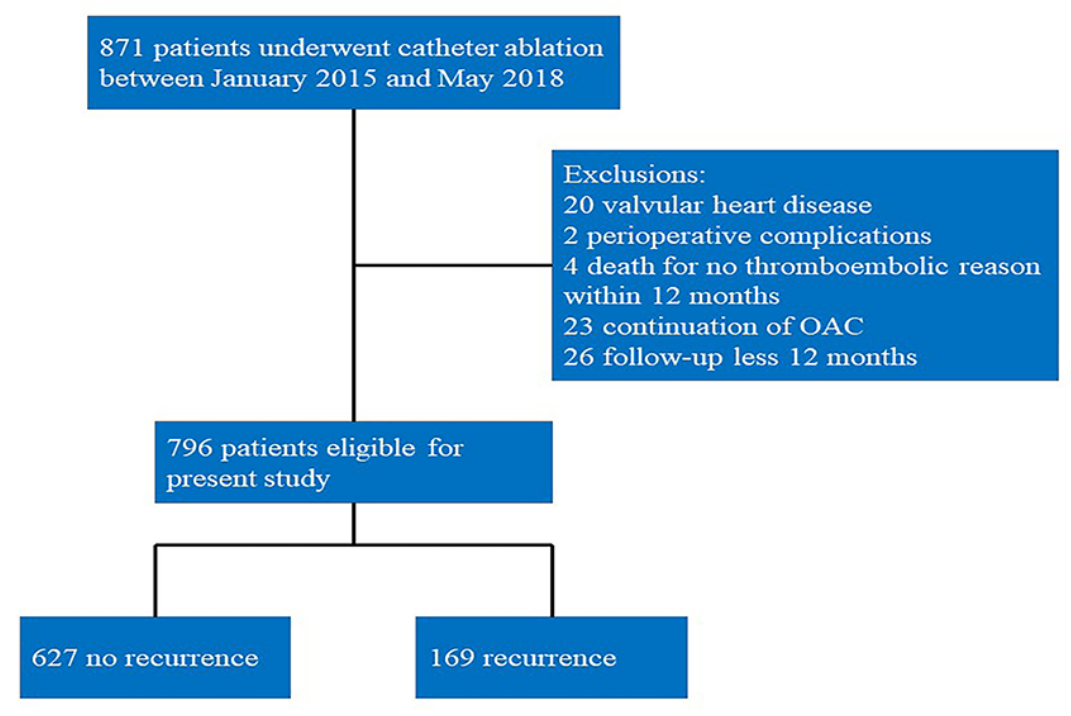


Figure 2
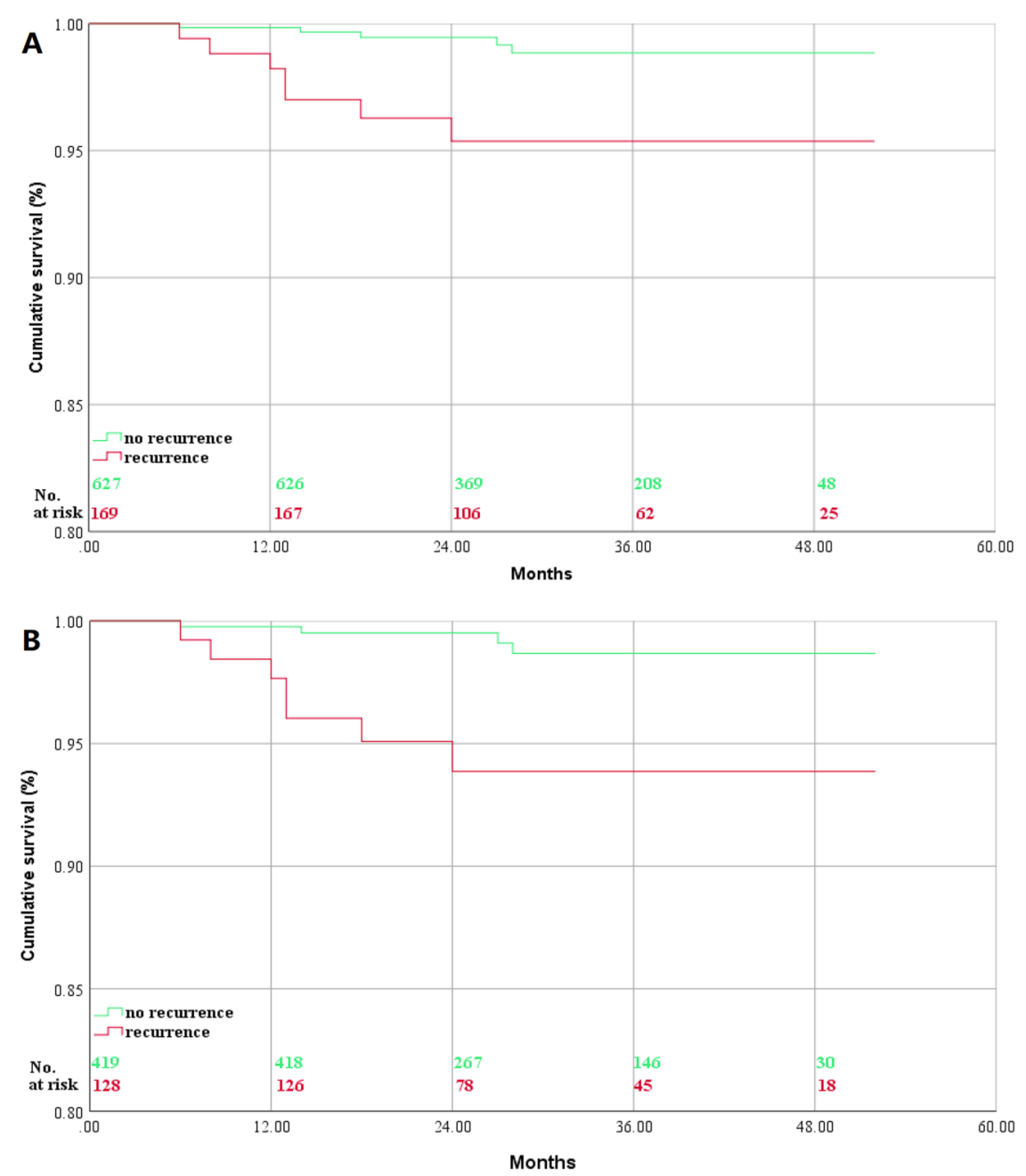